\title{
Broadband phase modulation by adiabatic pulses
}

\author{
Carlos A. Meriles, Dimitris Sakellariou, Alexander Pines* \\ Department of Chemistry, University of California and Materials Sciences \\ Division, Ernest Orlando Lawrence Berkeley National Laboratory \\ Berkeley, CA 94720, USA
}

\begin{abstract}
The use of inhomogeneous but spatially correlated static and radio-frequency (rf) magnetic fields conceptually offers a potential methodology for performing magnetic resonance spectroscopy of samples placed outside the bore of the magnet. However, its practical implementation still presents challenging problems, among them the control of nuclear spins over broad frequency offset intervals. The present study introduces an efficient method of encoding the phase of the magnetization when the variation of the static field along the sample is much larger than the $\mathrm{rf}$ amplitude. The procedure is based on the use of consecutively applied full-passage adiabatic pulses. The induced phase modulation is broadband and selective because it does not depend on the offset relative to the central frequency and the limits can be sharply defined. Finally, the encoded phase depends almost linearly on the local $\mathrm{rf}$ amplitude. All these features enable the recovery of an inhomogeneity-free spectrum with amplitudes close to the theoretically attainable maximum.
\end{abstract}

\footnotetext{
* To whom correspondence should be sent.
} 


\section{Introduction}

Current applications of Nuclear Magnetic Resonance (NMR) as a spectroscopic technique rely on having intense and homogeneous magnetic fields on extended samples. Slight spatial variations of the resonance frequency considerably affect the spectral resolution and, so far, this difficulty has restricted the use of NMR spectroscopy to samples inside well-shimmed solenoidal magnets. However, a broad set of applications is conceivable if a less demanding geometrical disposition could be used instead: wide open scanners or one-sided magnets able to provide spectroscopic information would be extremely useful in diverse areas from medicine to geology.

Recently, it has been shown that the simultaneous presence of static $\mathrm{B}_{0}$ field and rf gradients can be used to recover spectra in inhomogeneous fields. ${ }^{1}$ The technique is based on the use of composite $z$-rotation pulses that take advantage of the intrinsic inhomogeneity of the rf field to impose a spatial modulation on the phase of the local sample magnetization. If the static and rf fields are spatially correlated, the free evolution

after rf excitation and encoding gives rise to nutation echoes. ${ }^{2,3}$ In the idealized case, the echoes are not affected by the field inhomogeneities and depend only on the chemical shifts of the different nuclear spin components in the sample. If the procedure is periodically repeated to create a train of pulses and if the acquisition is restricted to the peaks of the subsequent echoes, the resulting FID leads to a high-resolution spectrum of the sample.

This technique poses significant challenges if "scanning spectroscopy" with onesided magnets is set as a goal. One of the main reasons is that the magnitude of the field gradient often imposes a variation along the sample many times larger than the amplitude 
of the rf field. The large offset implies that only the small fraction of the sample that is close to resonance can be properly encoded. As a result, the rapid reduction of the echo amplitude makes detection very difficult in practice. ${ }^{4}$ As shown below, this problem can be alleviated by the use of a double passage of adiabatic pulses sweeping along the frequency range of interest. Fig. (1) shows the induced modulation on one of the transverse components of the magnetization as a function of the resonance frequency and rf amplitude. The picture implies that the modulation is broadband in the sense that the effect is independent of the offset relative to the central frequency, even for very low rf fields: in this particular case, the ratio between the frequency bandwidth and the peak amplitude of the pulse reaches $\sim 20$ in the most unfavorable cases. At the same time, this process is selective in the sense that the modulated band can be defined in a precise manner. Finally, the dependence as a function of the rf field is almost linear, which ensures a reduced distortion of the refocusing in well correlated fields.

The proposed strategy is extremely simple and basically consists of two fullpassage adiabatic pulses applied immediately one after the other. Both pulses are identical with the exception of the peak amplitude whose relative variation determines the degree (and sense) of the modulation. The process taking place along the double passage can be clearly visualized from the double rotating reference frame. The first passage induces a modulation of the transverse magnetization that strongly depends on the local value of the rf field and, to a lesser extent, on the offset relative to the central frequency. In the double rotating frame, the beginning of the second passage is seen as an inversion of the effective field that, consequently, reverses the sense of the modulation. However, because the rf amplitude is different, the accumulated phase during the first passage is not 
completely canceled during the second one. The difference is roughly proportional to the local $\mathrm{rf}$ field value resulting in a modulation that reproduces its spatial variation. The offset dependence, already weak, is canceled out since the frequency sweep during the second passage is identical to the first one. This qualitative argument, holds to a good extent if the adiabatic passage has a hyperbolic secant dependence. ${ }^{5,6}$ However, smooth variations of the calculated pattern (Fig. (1)) have been observed if other adiabatic pulses common in the literature are used instead. ${ }^{7,8,9} \mathrm{We}$ return to this point later.

\section{Experiment and discussion}

The experimental performance of the proposed scheme was tested by means of an array that emulates the spatial dependence of the rf and static fields acting on a sample that faces a one-sided magnet. ${ }^{4} \mathrm{~A}$ thin glass cylinder filled with a mixture of acetone and benzene and immersed in the bore of a $4 \mathrm{~T}$ superconducting magnet, was irradiated by means of a conical coil. This creates an rf field that linearly decays along the sample. At the same time, a static field gradient along the coil axis was provided during the pulse sequence by means of an imaging setup.

Fig. (2A) shows the FID recorded in the presence of a $20 \mathrm{kHz} / \mathrm{cm}$ field gradient. A hard $90^{\circ}$ pulse was used to provide initial excitation, and a weak but long adiabatic double passage was applied immediately after to encode the phase of the sample magnetization. As explained above, the combined presence of matched rf and static field gradients results in a sharp nutation echo taking place at $1 \mathrm{~ms}$. The echo position can be controlled by the relative amplitude of each adiabatic full passage. A Fourier transform of this FID provides an rf-weighted image of the encoded phase (Fig. (2B)): as the offset 
frequency goes from positive to negative values, the rf amplitude diminishes accordingly. This translates into a change of the local phase of the magnetization and a corresponding modulation of the spectrum. For very low rf amplitudes, the adiabaticity condition is not fulfilled, leading to uncontrolled behavior. However, because the signal contribution is also very low from these points, it does not represent a serious distortion of the echo. For comparison, a non-modulated image obtained after a single pulse excitation is shown in the figure insert. As expected, a good correspondence between this last spectrum and the envelope of the modulated image is observed.

This same procedure can be repeated to create a train of adiabatic refocusing pulses. The evolution of the magnetization in the inter-pulse intervals along the train is shown in Fig. (2C). If the acquisition is restricted to the peaks of the echoes (open circles), the resulting FID is inhomogeneity-free and contains the full spectral information (Fig. (2D)). It is worth noting that the amplitude of the first echoes is comparable to the signal immediately following the excitation pulse indicating that essentially the whole sample constructively contributes to the echo. In the limit of high field gradients, this last point is particularly important: assuming an optimal excitation (obtained, for example, by broadband pulses such as BIR- $4^{10}$ ) the echo amplitude can approach its theoretically attainable maximum. The adiabatic phase modulation represents a considerable improvement over the performance of composite $z$-pulses where only a small fraction of the sample can be successfully refocused mainly due to limitations created by the offset (see Fig. (1)).

The gradual decay of the amplitude in subsequent echoes mainly derives from the repeated accumulation of errors in the refocusing; caused by an imperfect correspondence 
between the modulation induced by the pulses and that induced by the field gradient. This behavior could be anticipated in Fig. (1) where the modulation rate depends, albeit smoothly, on the amplitude of the rf field. Gradients perpendicular to the coil axis (not compensated for in our setup) and a natural decay due to relaxation and diffusion also represent potential sources of signal damping.

Despite all these limitations, the utility of a limited rf amplitude to control a broad frequency bandwidth for ex-situ cases emerges from our experiments: Fig. (2B) shows that the inhomogeneous broadening along the sample reaches $20 \mathrm{kHz}$ in the present example. This value is considerably higher than the $3 \mathrm{kHz}$ available during the peak of the adiabatic pulse in the region of maximum rf. It is also interesting to note that a series of spectra of decreasing resolution can be obtained with the same sequence if the amplitude of the second passage is altered beyond the minimum required in Fig. (2C). In this case, the echo occurs earlier and the stroboscopic acquisition in the center of the free evolution interval should provide free induction decays in the presence of effective gradients controlled by the rf field. Variable gradient imaging along the axis of dominant inhomogeneity is thus an attractive possibility, particularly for one-sided scanners where the field decay is a parameter not normally under experimental control.

Because the technique depends on the assumption that the static and rf fields are correlated, the sample spins can be imagined as forming a curve with positive slope in a frame similar to that of Fig. (1). If the modulation created by the pulse is strictly proportional to the local rf field, only a fraction of the sample is refocused when this curve is not a straight line. However, the spin response to the rf amplitude can itself be modified to encode a phase modulation proportional to the static field at each point. In 
such a case, the modulation can be altered if other parameters such as the central frequency or the bandwidth of the sweep are modified during the second passage. Further calculations have shown that the offset dependence is smoothly reintroduced in the selected frequency interval leading to either tilted or bent bands in Fig. (1). When projected onto the rf/static field correlation curve, this dependence now leads to an altered response of the local magnetization to the rf inhomogeneity. This kind of "active" field matching could enable the generalization of this technique to arbitrary rf profiles.

\section{Conclusion}

The methodology presented here offers a simple and efficient means to recover artifact-free spectra of almost optimal amplitude from samples in inhomogeneous magnetic fields. The adiabatic double passage encodes a phase modulation on the sample magnetization that depends almost linearly on the rf amplitude and is offset-insensitive, even in the case of weak rf fields. Assuming that the static and $\mathrm{rf}$ magnetic fields are linearly correlated along the sample, the free evolution following the double passage creates nutation echoes exhibiting an amplitude close to the maximum attainable. Experimental evidence shows that the lack of complete proportionality between the local rf amplitude and the induced phase shift leads to systematic errors that accelerate the damping of the echoes.

From a practical point of view, the problem can be set in the following terms: given an $\mathrm{rf}$ field profile, which is the $\mathrm{rf}$ pulse that induces a phase modulation proportional to the local static field? This "active" field matching still represents a challenge although the methodology presented here could provide a starting point. 
Since the adiabatic pulses are comparatively long, the process may prove inadequate for systems with short relaxation times (e.g., systems with paramagnetic species) or with a high diffusion coefficient (e.g., hyperpolarized gases). Also, the effect of J-couplings during the evolution under rf encoding can influence the spin dynamics and lead to distorted FIDs. The use of shorter numerically optimized pulses could offer a potential solution to these problems. ${ }^{11}$

Finally, it is worth mentioning that, given the offset-free control on the induced phase modulation, other techniques could also benefit from the adiabatic double passage scheme. As briefly mentioned, magnetic resonance imaging by radiofrequency gradients $^{12}$ is one example but other applications such as the suppression of unwanted signals ${ }^{13}$ or the manipulation of residual dipolar fields ${ }^{14}$ are possible as well.

\section{Acknowledgment}

This work was supported by the Director, Office of Science, Office of Basic Energy Sciences, Materials Sciences and Engineering Division, of the U.S. Department of Energy under Contract No. DE-AC03-76SF00098.

\section{References}

${ }^{1}$ CA Meriles, D Sakellariou, H Heise, A Moule, A Pines, Approach to high resolution ex-situ NMR, Science 293, 82 (2001)

${ }^{2}$ A Jerschow, Multiple echoes initiated by a single radio frequency pulse in NMR, Chem. Phys. Lett. 296, 466 (1998). 
${ }^{3}$ A Sharfenecker, I Ardelean, R Kimmich, Diffusion measurements with the aid of nutation spin echoes appearing after two inhomogeneous radiofrequency pulses in inhomogeneous magnetic fields, J. Magn. Reson. 148, 363 (2001).

${ }^{4}$ D Sakellariou, CA Meriles, A Moule, A Pines, Variable rotation composite pulses for high resolution nuclear magnetic resonance using inhomogeneous magnetic and radiofrequency fields $J$. Chem Phys. 363, 25 (2002), doi:

${ }^{5}$ J Baum, R Tycko, A Pines, Broad-band and adiabatic inversion of a 2-level system by phase modulated pulses, Phys. Rev. B. 32, 3435 (1985).

${ }^{6}$ MS Silver, RI Joseph, DI Hoult, Selective spin inversion in Nuclear Magnetic Resonance and Coherent Optics through an exact solution of the Bloch-Ricatti equation, Phys. Rev. A 31, 2753 (1985).

${ }^{7}$ E Kupče, R Freeman, Optimized adiabatic pulses for wideband spin inversion, J. Magn. Reson. Ser A 118, 299 (1996).

${ }^{8}$ RA DeGraaf, K Nicolay, Adiabatic rf pulses: Applications to in vivo NMR, Concept Magnetic Res. 9, 247 (1997).

${ }^{9}$ DG Norris, Adiabatic radiofrequency pulse forms in biomedical nuclear magnetic resonance, Concept Magnetic Res. 14, 89 (2002).

${ }^{10}$ M. Garwood, K Yong, Symmetrical pulses to induce arbitrary flip angles with compensation for $\mathrm{rf}$ inhomogeneity and resonance offsets, J. Magn. Reson. 94, 511 (1991).

${ }^{11}$ MA Smith, H Hu, AJ Shaka, Improved broadband inversion performance for NMR in liquids, J. Magn. Reson. 151, 269 (2001).

${ }^{12}$ D Canet, Radiofrequency field gradient experiments, Prog. Nucl. Mag. Res Sp. 30, 101 (1997).

${ }^{13}$ WE Maas, DG Cory, Heteronuclear correlation spectroscopy with $\mathrm{rf}$ gradients, $J$. Magn. Reson. A 112, 229 (1995).

${ }^{14} \mathrm{~S}$ Vathyam, S Lee, WS Warren, Homogeneous NMR spectra in inhomogeneous fields, Science 272, 92 (1996). 


\section{Figure captions}

Figure 1: Modulation of the transverse component $M_{x}$ of the magnetization in a sample of uncoupled spins after a double adiabatic passage as a function of the offset (relative to the central frequency of the sweep) and the peak amplitude of the pulse. In this case, $8 \mathrm{~ms}$ hyperbolic secant pulses were used and the frequency bandwidth was $100 \mathrm{kHz}$. The peak amplitude in the region of maximum rf amplitude was $20 \mathrm{kHz}$ and this value was reduced $50 \%$ during the second passage. The induced phase is offset independent and is almost proportional to the rf. For low enough rf amplitude, the adiabaticity condition is not properly fulfilled and the modulation is compromised. In the same way, the control vanishes outside the selected frequency bandwidth. As a reference, the central light square approximately represents the region controlled by means of square composite $z$ pulses when these are used to induce a phase modulation. For this latter case, it has been assumed that the amplitude is the peak amplitude used in the adiabatic pulse and that they are all applied at the central frequency of the bandwidth.

Figure 2: (A) Nutation echo from an acetone/benzene mixture induced after a single pulse excitation (10 $\mu$ s hard $\pi / 2,20 \mathrm{kHz}$ maximum rf) immediately followed by an adiabatic double passage. Each full passage was a 20 ms hyperbolic secant pulse and the amplitude ratio between the first and the second passage was 0.5 . The peak rf power was $3 \mathrm{kHz}$ whereas the static field gradient (present during and after the pulses) was 20 $\mathrm{kHz} / \mathrm{cm}$. (B) Fourier transform of Fig. (2A). The adiabatic double passage encodes a rf dependent phase shift. During the acquisition, the static field gradient makes it possible to image the encoded phase along the sample. The rapid and uncontrolled modulation at low 
frequencies (corresponding to low rf values) results from the lack of adiabaticity in this part of the sample. Due to the conical shape of the coil, the envelope corresponds to the the rf-weighted image obtained after a single pulse excitation (insert). (C) Free induction signal immediately after the excitation pulse and in subsequent free evolution periods after each double passage of the pulse train. The field gradient used was $20 \mathrm{kHz} / \mathrm{cm}$ and the pulses where $10 \mathrm{~ms}$ hyperbolic secant type. The amplitude ratio (0.7:1) is adjusted so that the echoes take place in the middle of the free evolution interval. (D) If only the echo centers are stroboscopically acquired (open circles in fig. (2C)) the effective FID maintains the modulation induced by the different chemical shifts. After a Fourier transform, the resulting spectrum is free of inhomogeneities and is comparable to the unperturbed spectrum recorded in the absence of field gradients (insert). 
Fig. 1: Meriles et al.

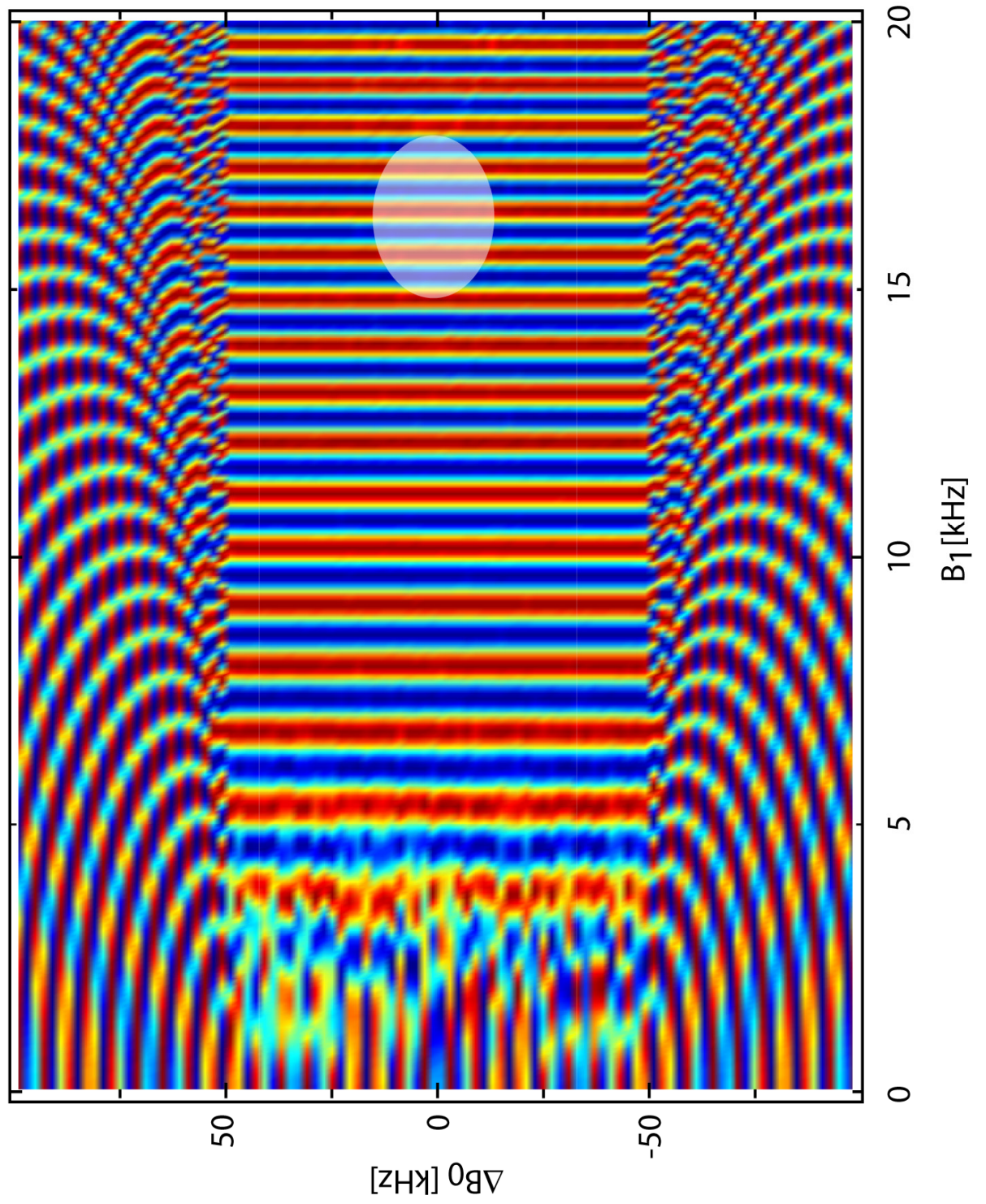


Fig. 2: Meriles et al.
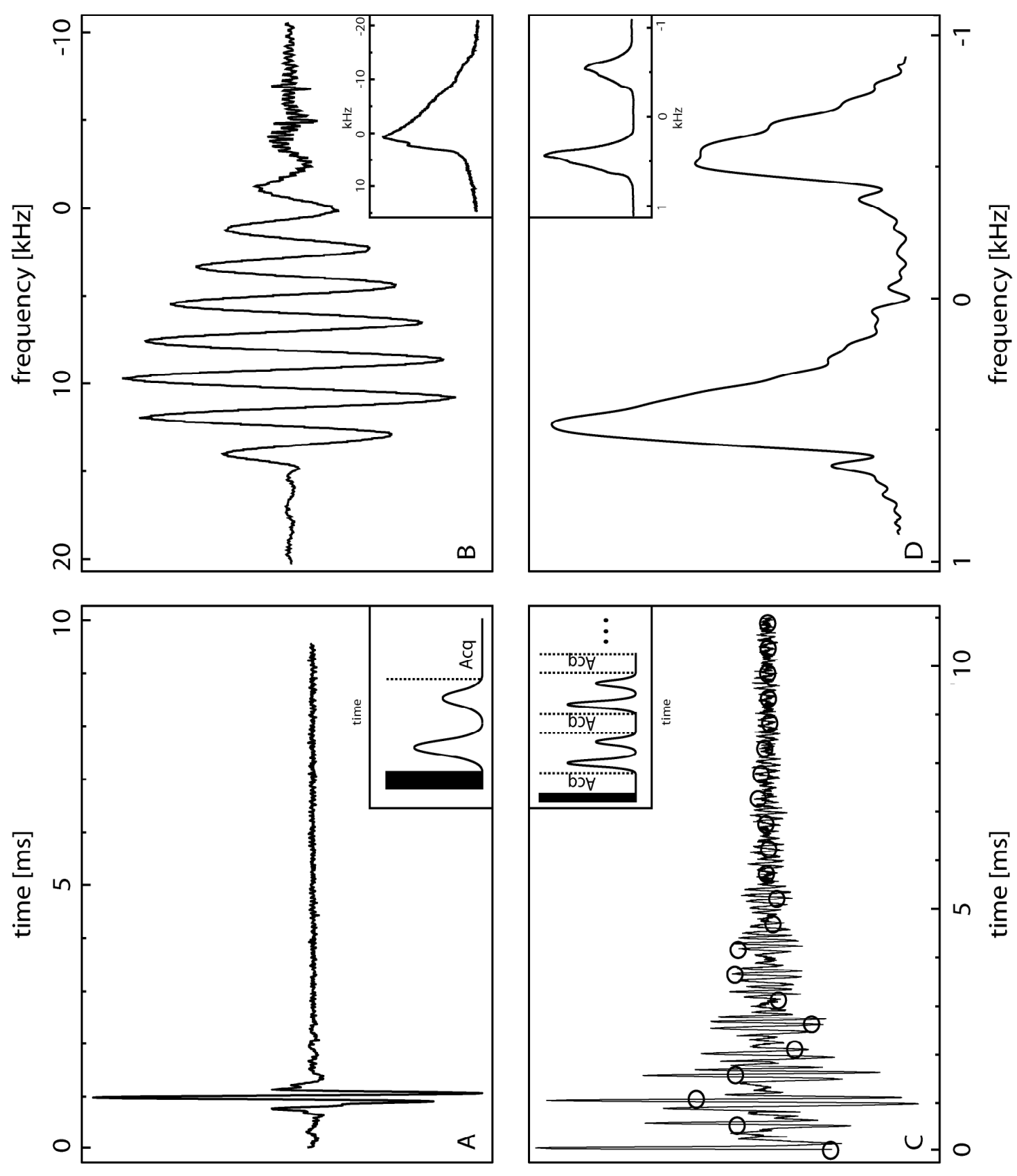